Session

\title{
Animating Mechanical Testing
}

\author{
Michael J. Kozak, Frank K. Brattain \\ Purdue University School of Technology
}

\begin{abstract}
For illustrative purposes it was desired to have a dynamic image showing how a tensile test specimen reacted when loaded in tension to failure. This paper describes the methodology used by the authors to create images based on surface data gathered during a tensile pull test. The data was gathered using a jointed spherical geometry digitizer. Interface software was utilized to capture the digitizer data and to transfer the data to the modeling software package. The captured data was used to create images representing the test data. Manipulation of the data to create the images is described. The images were then animated to give the appearance of a continuous dynamic test. The method used to create this effect is detailed. The authors examine possible improvements to the method used and suggest other possible applications for the technique developed. The animation is used in strength of material classes for the purpose of analyzing mechanical failures of components subjected to severe tensile loading conditions. The animation allows the mechanics of the failure to be demonstrated in detail.
\end{abstract}

Introduction

Tensile tests are often used to determine the yield strength and ultimate strength of a material ${ }^{1}$. A tensile test is often used as a demonstration of material property determination in a strength of materials class. Viewing a tensile test from start to finish makes for an interesting demonstration of tensile fracture and failure of a material specimen. The failure of the specimen can then be extrapolated by use of example to other mechanical members. This can help the student gain a feeling for the mechanism of tensile failure in mechanical components.

A tensile test is performed as one of the initial laboratory exercises in strength of materials class at the authors' location. Students gather around the universal testing machine (UTM) as a specimen is pulled to failure. It can be difficult for the students to observe the test. The specimen is fairly small and deformations may not be easily noticed. The observer must also have a desirable vantage point from which to observe the test. There is usually not room for more than a few students to have a good viewing location. Even with a favorable viewpoint the subtleties of the deformation may not be perceived by the novice.

Unfortunately, the end result of the tensile test usually consists of the initial sample dimensions, the fractured part, and pull force versus length information. The authors wanted to capture the 
elongation and fracture of the specimen as a dynamic visual image so it might be replayed as an example of how mechanical components react when subjected to an increasing tensile force until fracture occurs. The authors decided to capture 2-D digital information of the specimen in various stages of elongation until fracture and then use the captured digital data to create a sequence of 2-D images that could then be used to animate the test from start to finish.

\section{Capturing Data}

The experimental setup, shown in figure 1, consisted of a universal testing machine, tensile pull specimen, dial indicator affixed to the lower UTM platen and monitoring the movement of the upper UTM platen, a Microscribe model G-2 digitizer mounted to a large tripod in front of the platens, and an operator. The digitizer is a spherically jointed articulated $\operatorname{arm}^{2}$ which has a resolver at each joint, and software to calculate the location of the arm's tip in space ${ }^{3}$. The digitizer captures digital dimensional data by the operator placing the tip of the stylus at the location of the position desired, and then pressing a rocker switch. By holding the rocker switch down continuously while moving the stylus, a series of point locations were captured digitally. The authors used this continuous method of obtaining data at approximately 0.040 inch intervals. The continuous capture interval distance is set by the user via the Microscribe data capture software. The digitizer reference axis were defined by capturing three reference points. The authors used the upper left and both lower specimen corners as axis reference points.

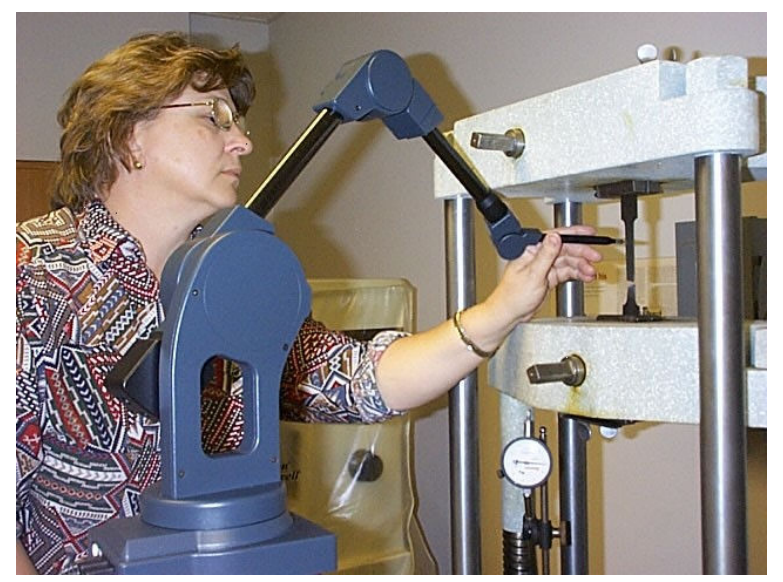

Figure 1. Capturing Digital Data

Two sets of dimensional data were captured at each step of elongation. One set down the left, and one down the right side of the specimen, each beginning at the upper corner where the specimen begins to neck down. Initially, the specimen was recorded with the stylus before a tensile load was applied. Then the specimen left and right sides were recorded as a pair after approximately every 0.200 inch movement of the upper platen with respect to the lower platen as shown by the dial indicator gage. This resulted in eight data set collections, the last one being the specimen after fracture. It was difficult to traverse the edge of the specimen without gathering extraneous data when the stylus inadvertently lost contact with the edge of the specimen. Data gathering became more reliable when a flat surface was placed against the back 
of the test specimen during stylus tracings. The stylus tip could then be constrained against the backing plate in the $\mathrm{z}$ axis (depth) direction. This removed one degree of freedom from stylus tip and greatly reduced the need to redo traces.

\section{Image Creation and Animation}

Each of the eight sets of data were then used to make a unique replication of the specimen's geometry in a 2-D image form. The 2-D images were created in Rhinoceros version 3.0 using the data files created in the Microscribe data capture files to serve as point clouds. The data capture software (Microscribe Utility Software, [MUS]) allows the user to save the information in an Excel file ${ }^{4}$. This Excel file was then imported to the Rhinoceros software. The resulting file is used to create a point cloud ${ }^{4}$. The corner points of the beginning of the narrowing of the unloaded sample were used as control points for a non-rational Bezier spline (NURBS) curve in the Rhinoceros software package. Poly lines were added horizontally to join the specimen's left and right side data runs at the top and bottom. The image was then joined into a closed contour and extruded into the initial thickness of the sample. The eight images could then be shown on the computer screen rapidly to form a dynamic sequence showing the captured states of elongation and rupture. The authors used fading of sequential images to enhance the transition between images.

\section{Resulting Images}

The first image, the leftmost in figure 2 , is the test specimen in its original condition before a tensile load is applied. The next four images show the elongation and narrowing of the specimen. The sixth image shows the beginning of a crack on the left side of the specimen. The seventh image shows the crack propagating. The eighth image shows the sample just before complete rupture.

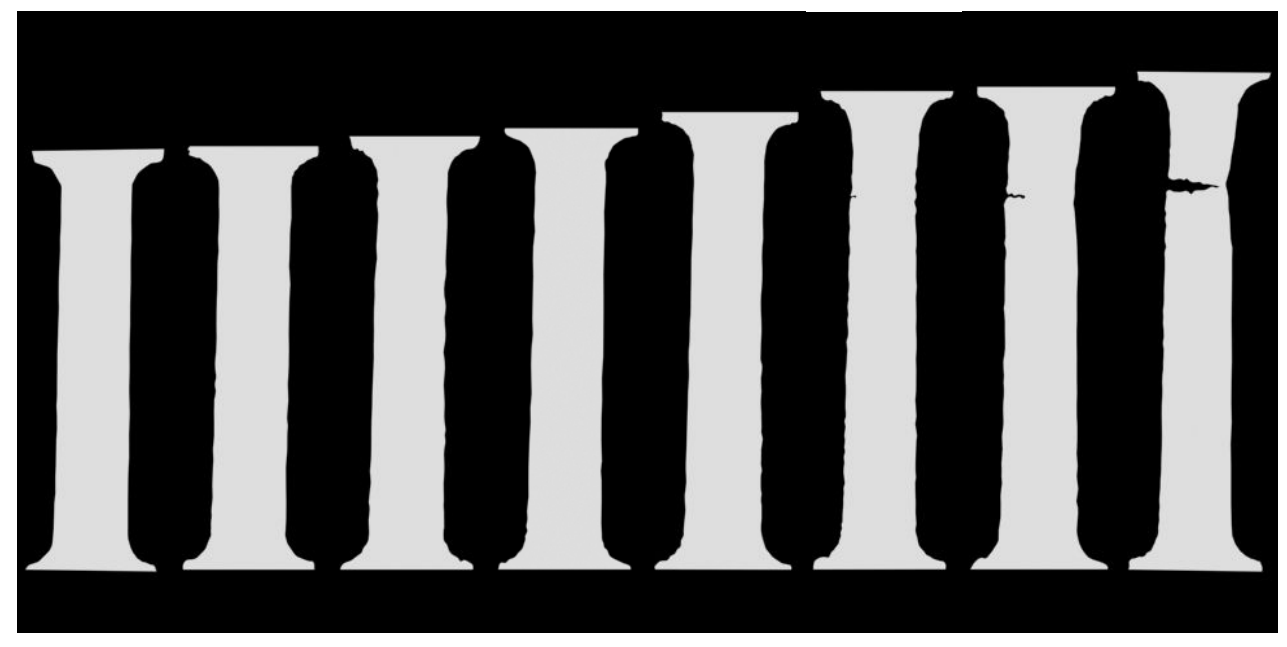

Figure 2. Resulting Images 
The axis relating to the depth of the sample (the $\mathrm{z}$ axis) was superfluous in our case since we only used the $\mathrm{x}$ and $\mathrm{y}$ dimensions to create the images. The final digital model was in three dimensions but the depth was forced to a uniform 0.040 inch thickness which was not representative of the actual specimen thickness as it was deformed. Traces along the front and rear face of the specimen could be gathered and representative three dimensional models could be created. This was beyond the scope of the initial animation project.

\section{Measurements from Data Files}

Making measurements of the specimen from the digital data can be accomplished by working with the raw data file that was captured in Excel (in this case). For example, if we want to measure the width of the specimen at some horizontal plane we can examine the excel data file and compare the dimension (the $\mathrm{x}$ dimension in this case) relating to the width in the raw data file. The data is captured in a format with $\mathrm{x}, \mathrm{y}$, and $\mathrm{z}$ coordinate data strings for each side of specimen.

\section{Morphing Images}

Images could be further animated by the digitally merging of sequential images (morphing) which would represent the intermediate stages of elongation which were not captured. The software package used by the authors to create the images had morphing capability. A difficulty with the package was the requirement that the images to be morphed contain the same number of vertices (data points). It was also desirable that the corresponding data points from image to image line up in one of the axis. The data that was gathered to form the images did not contain the same number of vertices or line up with respect to one of the axes. The images could therefore not be morphed without data manipulation. The data points were gathered by tracing along the edge of the specimen and capturing a new data point every time the digitizer stylus moved approximately $0.040 \mathrm{inch}$. As the specimen was stretched the number of data points grew. This was one cause of the number of vertices varying from data run to data run. The digitizer was not consistent in capturing data in 0.040 inch intervals. The distance between captured points seemed to vary depending on the speed at which the stylus traversed the surface. This was another cause of data point quantity variation between runs. As the specimen tore there was an increase in surface edge lineage to traverse. This was yet another cause of unequal data points between runs.

\section{Other Applications}

The digitizer could be used in various other mechanical tests to replay test images or make test measurements from the data files. Deflections of mechanical members or structures could be captured by the digitizer. Deformations due to impact could easily be gathered. The digitizer could also capture dynamic motion by affixing the stylus to the object in motion.

\section{Student Reaction}

Students were shown an animation of the images in figure 2 after having seen a live tensile test performed earlier in the semester. Students remarked that their memory of the live test had 
faded. They also commented that they noticed details in repetitive playing of the animation that they did not notice in the live test. Furthermore, students commented that it was much easier to focus on the specimen distortion when isolated from the distraction of the testing equipment. Also, every student in the class had an optimal viewing advantage as the images are displayed on their computer screen or as a projected image on a screen. This is compared to students craning their necks and competing with each other to get within the limited viewing area available during a real time tensile test. The display of the images also allows the magnification of the specimen size as opposed to students trying to notice small changes to a small specimen.

\section{Conclusion}

The sequence of images was then shown as an aid to the discussion of how materials fail in the tensile mode. Images can be created for ductile materials and compared to images of brittle materials. Captured images can be replayed and dissected, whereas simply observing a tensile test and trying to commit the sequence of material deformation during elongation and fracturing to memory is prone to error and/or loss.

Capturing the image in an electronic format allows the instructor to easily demonstrate to the students the sequence of events during a pull test. Displaying the electronic images is easily accomplished compared to performing the actual pull test. The electronic images can also be viewed at locations where the mechanical pull test equipment is not available.

\section{Bibliography}

1. Robert L. Mott, Applied Strength of Materials, $4^{\text {th }}$ edition, Prentice Hall, New Jersey, 2002

2. James A Rehg, Introduction to Robotics in CIM Systems, $2^{\text {nd }}$ edition, Prentice Hall, New Jersey, 1992

3. Microscribe G-2 Users Manual, Immersion Corporation

4. Microscribe Utility Software Manual, Immersion Corporation 